ks. Michael Kahle

\title{
Wie Maria heute ehren?
}

Die titelgebende Frage „Wie Maria heute ehren?" gehört „sicher nicht zu denen, die den Menschen heute das meiste Kopfzerbrechen bereiten" ", besonders wenn man bedenkt, dass die Kirche vor allem in der westlichen Welt angesichts der Missbrauchsskandale, des Zusammenpralls der Kulturen und Religionen, der fortschreitenden Säkularisierung etc. vor ganz andere Herausforderungen gestellt und mit ganz anderen Themen konfrontiert ist. Dennoch gehört die oben vorgetragene Frage zu den zentralen Punkten, um zu einem vor Gott verantworteten Glauben zu kommen, denn die Antwort ist auch ein Hinweis darauf, ob der Christ im wahren Glauben der Kirche verortet und mit Gott wahrhaft verbunden ist.

\section{Die Volksfrömmigkeit und die Liturgie}

Der Weg zu einer Antwort setzt beim Zweiten Vatikanischen Konzil ein und führt über den heiligen Papst Paul VI. in das Pontifikat des heiligen Papstes Johannes Paul II.: Während der große Sohn des polnischen Volkes als Stellvertreter Jesu Christi auf Erden und Nachfolger des Apostelfürsten Petrus die Kirche

1 W. Beinert, Einführung zur Enzyklika Marialis Cultus, Leutesdorf 1974, 5. (Kurzform: Beinert) 
sicher durch die Zeit führt, erarbeitet die Kongregation für den Gottesdienst und die Sakramentenordnung im großen Jubiläumsjahr 2000 das Direktorium über die Volksfrömmigkeit und die Liturgie. ${ }^{2}$ Dieses Dokument wurde, nach der Approbation durch Papst Johannes Paul II., mit Dekret vom 17. Dezember 2001 veröffentlicht. ${ }^{3}$ Das Direktorium gibt vor allem die verbindliche kirchliche Antwort auf die Frage nach der Ausdrucksform der Marienverehrung bzw. des „Cultus Marialis“ für die Kirche im dritten Jahrtausend.

Bevor jedoch genauer auf eine glaubens- und zeitgemäße Marienverehrung eingegangen wird, bedarf es eines Blickes auf den grundsätzlichen Zusammenhang von Liturgie und Volksfrömmigkeit, denn der Cultus Marialis erstreckt sich, wie sich zeigen wird, sowohl auf die heilige Liturgie der Kirche wie auf die Volksfrömmigkeit.

Eine diesbezügliche Unterscheidung und Verhältnisbestimmung wird im ersten Teil des Direktoriums über die Volksfrömmigkeit und die Liturgie anhand leitender Prinzipien vorgenommen. Grundlegend gilt, dass die Liturgie und die Volksfrömmigkeit zwei Ausdrucksweisen des einen Kultes sind. Dabei ist die Liturgie, wie sowohl am Anfang des Direktoriums als auch am Anfang des Dekretes zu seiner Inkraftsetzung, mit Rückgriff auf die Liturgiekonstitution des Zweiten Vatikanischen Konzils Sacrosanctum Concilium ${ }^{4}$ herausgestellt wird, „der Höhepunkt, dem das Tun der Kirche zustrebt und zugleich die Quelle, aus der

2 Kongregation für den Gottesdienst und die Sakramentenordnung, Direktorium über die Volksfrömmigkeit und die Liturgie. Grundsätze und Orientierungen, vom 17. Dezember 2001 (VApS 160), Bonn 2001. (Kurzform: DVL)

3 Kongregation für den Gottesdienst und die Sakramentenordnung, Dekret Prot. N. 1532/00/L, 17. Dezember 2001, in: Notitiae 38 (2002), 464-465.

4 Zweites Vatikanisches Konzil, Sacrosanctum Concilium, in: H. Denziger, Kompendium der Glaubensbekenntnisse und kirchlichen Lehrentscheidungen, Freiburg ${ }^{44} 2014$, 4001-4048. 
all ihre Kraft strömt" (SC 10). In der Liturgie setzt Jesus Christus als Erlöser und Hoherpriester in seiner Kirche, mit ihr und durch sie das Werk Erlösung fort. Aus diesem Faktum rührt die Vorrangstellung der Liturgie gegenüber der Volksfrömmigkeit. Die Liturgie ist für das Leben des Menschen in Jesus Christus im Blick auf sein Heil notwendig. Die Formen der Volksfrömmigkeit hingegen haben für das Leben des Glaubenden fakultativen Charakter, auch wenn sie - wie sich an der Marienverehrung zeigen wird - äußerst nützlich und fruchtbringend sind. ${ }^{5}$ Aus der angeführten Unterscheidung ergibt sich, dass die Liturgie der Volksfrömmigkeit objektiv übergeordnet bzw. die Volksfrömmigkeit objektiv der Liturgie untergeordnet ist. ${ }^{6}$ Keinesfalls jedoch stellt die Volksfrömmigkeit zur Liturgie einen Gegensatz ${ }^{7}$ dar, denn sie „richtet ihr Augenmerk bevorzugt auf die Mysterien des Sohnes Gottes" (DVL 62). So ist die Volksfrömmigkeit, wie das Direktorium herausstellt, im Blick auf Jesus Christus „zuinnerst bewegt beim Bericht seiner Geburt und betrachtet die unendliche Liebe, die von diesem Kind ausgeht, das wahrer Gott und wahrhaft unser Bruder ist, arm und verfolgt seit seiner Kindheit. Sie hört gern die zahlreichen Szenen des öffentlichen Lebens unseres Herrn Jesus, der als guter Hirt Zöllnern und Sündern nahe ist, der als Wundertäter, Kranke heilt und Notleidenden hilft, der als Lehrer die Wahrheit spricht. Vor allem liebt sie es, die Geheimnisse seines Leidens zu betrachten, denn darin findet sie seine unendliche Liebe und seine große Solidarität mit menschlichen Leiden: Der verratene und verlassene Jesus, gegeißelt und mit Dornen gekrönt, gekreuzigt von Übeltätern, vom Kreuz abgenommen, im Schoß der Erde, beweint von Jüngern und Freunden" (DVL 79) steht im Zentrum der Volksfrömmigkeit. Außerdem nimmt das Verlangen nach Gemeinschaft mit den Bewohnern des Himmels, der seligen Jungfrau Maria, den

\footnotetext{
Vgl. DVL 11.

6 Vgl. ebd., 50.

7 Vgl. ebd.
} 
Engeln und Heiligen breiten Raum ein. ${ }^{8}$ Inhaltlich betrachtet steht die Volksfrömmigkeit also in einem engen Zusammenhang zur Liturgie, ja sie steht sogar in einem fruchtbaren Miteinander - als Ausdruck des einen Kultes - mit der Liturgie in Beziehung. ${ }^{9}$

\section{Die Verehrung der heiligen Mutter des Herrn}

Vor allem Kapitel V des Direktoriums über die Volksfrömmigkeit und die Liturgie gibt Orientierung im Blick auf die Marienverehrung für die Gegenwart, indem es eine nähere Bestimmung des Cultus Marialis bietet.

Von besonderer Bedeutung ist bereits die dem Kapitel gegebene Überschrift: „Die Verehrung der heiligen Mutter des Herrn“. Anstelle des im Calendarium Romanum gebräuchlichen Beatae Mariae Virginis bzw. der am Anfang des Kapitels V verwendeten Bezeichnung „selige Jungfrau und Gottesmutter Maria“" ${ }^{10}$ wird Maria ganz ausdrücklich durch ihre Beziehung zu ihrem Sohn Jesus Christus charakterisiert. Hier findet also umgehend Anwendung, was im ersten Teil des Direktoriums grundgelegt wurde: Von Maria ausgehend richtet Marienverehrung ihr Augenmerk letztlich immer auf das Mysterium des Sohnes Gottes. ${ }^{11}$ In DVL 183 wird dem schließlich noch die erläuternde Aussage hinzugefügt, dass „die Verehrung der seligen Jungfrau und Gottesmutter Maria [...] dem Glauben und der Liebe des Volkes Gottes gegenüber Christus, dem Erlöser der Menschheit [entströmt]“ (DVL 183). Daher verwundert es nicht, dass im Direktorium die zentrale Aussage des Apostolischen Mahnschreibens Marialis cultus des heiligen Papstes Paul VI. wiedergegeben wird, dass

\footnotetext{
8 Vgl. ebd., 62.

9 Vgl. ebd., 58.

10 Vgl. ebd., 183.

11 Vgl. ebd., 62; 79.
} 
sich die marianischen Andachtsübungen im „Flussbett des einzigen Kultes“ befinden müssen, der „mit gutem Recht christlich genannt wird, weil er von Christus Ursprung und Wirksamkeit bezieht, in Christus den vollendeten Ausdruck findet und durch Christus im Geist zum Vater führt" (DVL 186; MC 24).

Grundsätzlich wird an den Cultus Marialis folgende Anforderung gestellt: [a] Er muss trinitarisch ausgerichtet sein. [b] Er muss eine christologische Komponente haben, welche die einzige und notwendige Mittlerschaft Christi betont und [c] zudem eine pneumatologische Dimension besitzen wie [d] einen ekklesiologischen Charakter haben. Außerdem muss er sich [e] auf der Grundlage der heiligen Überlieferung, beständig auf das geoffenbarte Wort Gottes in der Heiligen Schrift beziehen. Diese Bestimmung des Cultus Marialis besitzt Gültigkeit sowohl für die Liturgie als auch für die Volkfrömmigkeit, weil sich die Marienverehrung ja auf beide Bereiche erstreckt. Klar zeigt sich dies im Kapitel IV des Direktoriums, und zwar in den Artikeln 101-102; 112; 115; 120-123; 136-137;145; 147; 149; 151; 174; 180-181, sowie im Kapitel V in den Artikeln 187 bis 207. In diesen Abschnitten wird der Cultus Marialis zum einen im Bereich der von der Liturgie geprägten Zeit des Kirchenjahres bestimmt, in der sich der Raum für die Marienverehrung öffnet, zum anderen im Bereich der Volksfrömmigkeit, wo einige wesentliche und zentrale marianische Andachtsübungen verankert sind, die vom Lehramt der Kirche besonders empfohlen werden.

\section{Der Raum für den Cultus Marialis in der Liturgie des Kirchenjahres}

Im Bereich der Liturgie wird der Cultus Marialis in seinen Ausformungen durch die Feste des Calendarium Romanum geprägt. So öffnet sich bei zahlreichen Festzeiten und Tagen des Kirchenjahres Raum für die Marienverehrung: 
a) In der das jeweilige Kirchenjahr eröffnenden Zeit des Advents kommt der Cultus Marialis in der Feier der Liturgie in beispielhafter Weise zum Ausdruck, denn die Kirche preist in dieser Zeit „die Haltung des Glaubens und der Demut, in der Maria aus Nazareth ohne Zögern und radikal den Heilsplan Gottes" (DVL 101) angenommen hat. Zugleich hebt die Kirche die Bedeutung Mariens für die Gnadenereignisse hervor, die der Geburt des Erlösers vorausgegangen sind. ${ }^{12}$

Das Hochfestes der ohne Erbsünde empfangenen Jungfrau und Gottesmutter Maria im Advent ist der besondere Platz für den Cultus Marialis. Das Hochfest selbst muss inhaltlich betrachtet als „Vorbereitung auf die Geburt Jesu“ (DVL 101) gesehen werden, drückt sich doch darin die lange messianische Erwartung und die Prophezeiungen des Alten Testamentes im Blick auf das Kommen des Messias aus.

Insofern steht auch das Hochfest der Verkündigung des Herrn, das die Kirche am 25. März begeht, in enger Beziehung zum Cultus Marialis des Advents: An diesem Tag, der genau neun Monate vor dem Weihnachtsfest liegt, feiert die Kirche ja die Inkarnation des Sohnes Gottes im Schoß der Jungfrau Maria.

b) In der Weihnachtszeit selbst öffnet sich der Cultus Marialis vortrefflich durch das Hochfest der Gottesmutter Maria, am Oktavtag von Weihnachten, dem 1. Januar. In „herausragender Weise" (DVL 115) ist das Hochfest marianisch, denn an ihm erklingt der Lobpreis der Jungfrau über die Geburt ihres göttlichen Kindes.

Noch ganz mit dem Weihnachtsfestkreis verbunden, aber bereits außerhalb desselben angesiedelt, bietet das Fest der Darstellung des Herrn die Möglichkeit für die Marienverehrung. An diesem Tag steht wiederum die Demut Mariens im Vordergrund des Cultus Marialis, da die Jungfrau Maria die Geste des Reinigungsritus nach den Vorschriften des mosaischen Gesetztes

12 Vgl. ebd., 101. 
vollzieht. Die Marienverehrung ist an diesem Tag jedoch ganz gebunden an die christologische Dimension des Festgeheimnisses. Dieses drückt sich im „Zeichen Christi des ,Lichts der Welt““ (DVL 123) aus. Maria ist die erste Zeugin des Lichtes, das ihr Sohn ist.

c) In der Österlichen Bußzeit zeigen sich der gekreuzigte Christus und die schmerzhafte Jungfrau Maria, die untrennbar zusammen gehören. „Wie Christus ,Mann der Schmerzen' (Jes $53,3)$ ist, durch den Gott seinen Plan verwirklichte, ,alles zu versöhnen im Himmel und auf Erden, um Frieden zu stiften am Kreuz durch sein Blut' (Kol 1,20), so ist Maria ,Schmerzensfrau', die Gott mit seinem Sohn vereinen wollte als an seinem Leiden Anteil nehmende Mutter" (DVL 136). Der Schmerz der Jungfrau Maria liegt in der Ablehnung ihres Sohnes. So geht der Weg Mariens unaufhörlich mit dem Weg Jesu zusammen und es wird im Cultus Marialis „ständig und unbedingt auf das Geheimnis Christi des leidenden Gottesknechtes“ (DVL 137) verwiesen.

d) Im Triduum Sacrum schließlich hat durch den Planctus Marie (das Weinen Mariens, vgl. DVL 145), die Marienverehrung einen besonderen Platz. Maria beweint nicht nur den Tod ihres unschuldigen und heiligen Sohnes, sondern auch die Verirrung seines und ihres Volkes und die Sünde des ganzen Menschengeschlechtes. Vom Karfreitag herkommend, erhält die Marienverehrung am Karsamstag immer größeren Raum, denn im Verweilen der Mutter am Grab ihres Sohnes findet der Cultus Marialis einen herausragenden Platz. „Während der Leib ihres Sohnes im Grab ruht und seine Seele in das Reich des Todes hinabsteigt, um seinen Vorfahren die bevorstehende Befreiung aus dem Schattenreich zu verkünden, wartet die Jungfrau glaubend an den Sieg ihres Sohnes über den Tod und verkörpert dabei in vorwegnehmender Weise die Kirche" (DVL 147).

e) Am Ostersonntag setzt sich die Verbindung zwischen Maria und ihrem Sohn fort. So wie Maria in der Stunde der Schmerzen und des Todes mit ihrem Sohn verbunden war, so ist sie es 
auch in der Stunde der Auferstehung und des Jubels. ${ }^{13}$ Dies erstreckt sich über die gesamte Osterzeit. Auch in der Vollendung der österlichen Festzeit öffnet sich der Raum für den Cultus Marialis, wenn die Kirche auf das Pfingstfest zugeht und Maria im Kreis der um den Heiligen Geist flehenden Apostel weiß (vgl. Apg 1,13-14).

f) Im Nachhall des Osterfestkreises begeht die Kirche nach dem Hochfest des heiligen Herzens Jesu das Gedächtnis des unbefleckten Herzens Mariens. „Das Geheimnis des Herzens des Erlösers wirft ein Licht auf das [Herz der Mutter, die auch Gefährtin und Jüngerin ist, und spiegelt sich darin wider]. Wie das Fest des [H]eiligen Herzens die heilbringenden Geheimnisse Christi gleichsam zusammengefasst feiert und sie zu ihrer Quelle zurückführt, nämlich das Herz, so ist das Gedächtnis des unbefleckten Herzens Mariens Ausdruck der ,herzlichen' Gemeinschaft der Mutter mit dem Erlösungswerk ihres Sohnes: von der Menschwerdung bis zum Tod und zur Auferstehung, bis zum Geschenk des Geistes" (DVL 174) ist Maria mit ihrem Sohn verbunden.

g) Schließlich bietet sich mit dem Hochfest Mariä Aufnahme in den Himmel, das die Kirche am 15. August feiert, der Platz für den Cultus Marialis im Jahreskreis. Maria gibt Zeugnis von der ganzen Fülle des Heilswerkes ihres Sohnes; sie ist das Unterpfand der künftigen Teilhabe aller Glieder des mystischen Leibes an der österlichen Herrlichkeit ihres auferstandenen Sohnes; sie ist das endgültige Zeichen der Hoffnung für alle, die an Christus glauben, dass auch sie vollendet werden; und schließlich ist sie das endzeitliche Bild dessen, was die ganze Kirche sehnsüchtig erwartet und erhofft. ${ }^{14}$

h) Die im Römischen Generalkalender wie in den verschiedenen Partikularkalendern enthaltenen Marienfeste eröffnen jeweils auf ihre Weise im Laufe des Kirchenjahres den Raum

13 Vgl. ebd., 149.

14 Vgl. ebd., 180. 
für den Cultus Marialis. Dies geschieht gemäß der Ordnung des Calendarium Romanum am nichtgebotenen Gedenktag Unserer Lieben Frau von Lourdes (11. Februar), am nichtgebotenen Gedenktag Unserer Lieben Frau von Fatima (13. Mai), am Fest Maria Heimsuchung (31. Mai), am gebotenen Gedenktag Maria, Mutter der Kirche (Montag nach Pfingsten), am nichtgebotenen Gedenktag Unserer Lieben Frau auf dem Berge Karmel (16. Juli), am nichtgebotenen Gedenktag der Weihe der Basilika Santa Maria Maggiore in Rom (5. August), am gebotenen Gedenktag Maria Königin (21. August), am Fest Maria Geburt (8. September), am nichtgebotenen Gedenktag Maria Namen (12. September), am gebotenen Gedenktag des Gedächtnisses der Schmerzen Mariens (15. September), am gebotenen Gedenktag Unserer Lieben Frau vom Rosenkranz (7. Oktober), am gebotenen Gedenktag Unserer Lieben Frau von Jerusalem (21. November) sowie am nichtgebotenen Gedenktag Unserer Lieben Frau von Guadalupe (12. Dezember).

i) Zu den Zeiten des Liturgischen Jahres und den Marienfeiertagen gesellt sich das Gedächtnis der seligen Jungfrau Maria am Samstag. ${ }^{15}$ Jeweils als memoria ad libitum ist die Feier zu Ehren der seligen Jungfrau und Gottesmutter Maria möglich. ${ }^{16}$ Das dazu von Papst Johannes Paul II. im Jahr 1986 approbierte Messbuch de Beata Maria Virgine legt für die gesamte Zeit des Jahreskreises entsprechende Messformulare vor. ${ }^{17}$ Im Zentrum steht vor allem die Erinnerung an die Haltung Mariens, die als Mutter und Jüngerin, erfüllt mit einem starken Glauben und einer unverbrüchlichen Hoffnung, am "Großen Samstag“ (PM 36) allein am Grab ihres Sohnes wacht und seiner Auferste-

${ }^{15}$ Vgl. ebd., 188.

${ }^{16}$ Vgl. Kongregation für den Gottesdienst und die Sakramentenordnung, Messbuch. Sammlung von Marienmessen. Pastorale Einführung, Freiburg 1990, Nrn. 21, 34-36. (Kurzform: PM)

${ }^{17}$ Kongregation für den Gottesdienst und die Sakramentenordnung, Messbuch. Sammlung von Marienmessen, Freiburg 1990. 
hung entgegenharrt. Die Feier des Mariensamstags ist „Präludium und Einführung in die Feier des Sonntags" (DVL 188), jenes Ur-Festes, an dem die ganze Kirche das wöchentliche Gedächtnis der Auferstehung Jesu Christi begeht. ${ }^{18}$

j) Schließlich öffnet sich parallel zum liturgischen Jahr der Raum für den Cultus Marialis in der Praxis des "Marienmonats“ (DVL 190). Auf der nördlichen Erdhalbkugel wird dieser im Mai und auf der südlichen Erdhalbkugel zumeist im November begangen. Im Direktorium wird es nicht unterlassen, darauf aufmerksam zu machen, dass diese Praxis nach wie vor „einige liturgie-pastorale Probleme" (DVL 190) mit sich bringt.

k) Unbeschadet dieser kritischen Sicht bietet zudem der Monat Oktober, der sich als Rosenkranzmonat durch die tägliche Praxis desselben auszeichnet, die Möglichkeit für die Marienverehrung. ${ }^{19}$

So ergibt sich, innerhalb des liturgischen Jahres unaufhörlich die Möglichkeit für den Cultus Marialis in der Liturgie. Die Kirche lädt damit die Gläubigen ein, „die Mysterien Christi in demselben Geist der Frömmigkeit zu feiern, in dem Maria selbst Anteil [daran] hatte" (PM 17).

\section{Vom Lehramt empfohlenen marianischen Andachtsübungen als Ausdruck des Cultus Marialis}

Im Direktorium über die Volksfrömmigkeit und die Liturgie werden einige marianische Andachtsformen aufgeführt, durch die der Cultus Marialis zum Ausdruck kommt.

a) Vom Lehramt der Kirche empfohlen wird das Gebet des Angelus während des Jahres bzw. des Regina Caeli in der Osterzeit. Beide Gebete bringen als marianische Andachtsform des Tages

\footnotetext{
18 Vgl. PM 36.

19 Vgl. DVL 198.
} 
am Morgen, am Mittag und am Abend den Cultus Marialis zum Ausdruck.

Geschichtlich betrachtet ist das Angelusgebet von Papst Pius V. in der Kirche eingeführt worden. ${ }^{20}$ Es erinnert an das große Heilsereignis, dass „nach dem Plan des Vaters das Wort durch den Heiligen Geist im Schoß der Jungfrau Maria Mensch geworden ist" (DVL 195). In diesem Gebet ist, auf biblischem Grund stehend, das Gedächtnis des ganzen Heilswerks und besonders der Inkarnation Gottes enthalten. ${ }^{21}$ Somit ist es ganz auf das trinitarische Geheimnis ausgerichtet und betont die besondere Bedeutung Jesu Christi. Außerdem zeichnet es sich durch eine pneumatologische wie ekklesiologische Dimension aus.

Ebenso theologisch zu charakterisieren ist die Antiphon des Regina Caeli, die am 20. April 1742 von Papst Benedikt XIV. als Gebet anstelle des Angelus für die Osterzeit angeordnet wurde. ${ }^{22}$ Gemäß dem Handbuch der Ablässe ${ }^{23}$ gewährt der Apostolische Stuhl für diese Gebete einen Teilablass. ${ }^{24}$

b) Das Rosenkranzgebet, auch „Psalterium der Jungfrau Maria“ genannt, zählt zu den hervorragendsten marianischen Andachtsformen, in denen der Cultus Marialis zum Ausdruck kommt. Papst Pius V. legte 1569 mit dem Breve Consueverunt Romani Pontifices ${ }^{25}$ die heute immer noch gültige Form des Rosenkranzgebetes fest. Diese Andachtsform ist in zahlreichen Apostolischen Schreiben und Enzykliken von den Päpsten the-

${ }^{20}$ Vgl. A. Heinz, Angelus, in: LThK3, Bd. 1, 654. (Kurzform: Heinz, Angelus)

21 Vgl. A. Heinz, „Der Engel des Herrn“. Erlösungsgedächtnis als Volksgebet, in: HlD 33 (1979), 51-58.

22 Vgl. Heinz, Angelus, 654.

23 Apostolische Pönitentiarie, Handbuch der Ablässe, Normen und Gewährungen, Vatikan 22008. (Kurzform: HdA)

${ }^{24}$ Vgl. HdA 17 §2, $2^{\circ}$.

25 Vgl. Pius V., Consueverunt Romani Pontifices, 17. September 1569, in: Bull. Ord. Praed., Bd. V, 223. 
matisiert und den Gläubigen empfohlen worden. ${ }^{26}$ Theologisch betrachtet ist es ein Gebet mit zutiefst biblischem Charakter, da in ihm sich „die Betrachtung der heilbringenden Ereignisse des Lebens Jesu Christi“ vollzieht, „mit denen die jungfräuliche Mutter eng vereinigt war" (DVL 197). In der jeweiligen Clausu$l a^{27}$, wird, „treu zu Schrift und Liturgie“ (DVL 201), das entsprechende Christusmysterium eingefügt.

Die außerordentliche Hochachtung vor dem Rosenkranzgebet kommt neben den zahlreichen empfehlenden Worten des Lehramtes, in zwei Punkten zum Ausdruck: zum einen durch den vorgesehenen Ritus der Segnung des Rosenkränzes ${ }^{28}$ und zum anderen durch die Gewährung des Ablasses für das Gebet des Rosenkranzes. Der Apostolische Stuhl gewährt nämlich, gemäß der bestehenden Bestimmungen, jedem Christgläubigen einen vollkommenen Ablass ${ }^{29}$ oder einen Teilablass. ${ }^{30}$

${ }^{26}$ Vgl. Leo XIII, Supremi apostolatus officio, 1. September 1883; Ders., Salutaris ille, 24. Dezember 1883; Ders., Superiore anno, 30. August 1884; Ders., Octobri mense, 22. Oktober 1891; Ders., Magnae Dei matris, 8. September 1892; Ders., Laetitiae sanctae, 8. September 1893; Ders., Lucenda semper expectatione, 8. September 1894; Ders., Fidentem piumque animum, 20. September 1896; Ders., Vi è ben noto, 20. September 1887; Ders., Augustissimae Virginis Marie, 12. September 1897; Ders., Diuturni temporis, 5. September 1898; Ders., Parta humano generi, 8. September 1901; Pius XI., Ingravescentibus malis, 29. September 1937; Pius XII., Ingruentium malorum, 15. September 1951; Johannes XXIII., Grata recordatio, 26. September 1959; Ders., Il Religioso convegno, 29. September 1961; Paul VI., Christi matri rosarii, 15. September 1966; Ders., Recurrens mensis october, 7. Oktober 1969; Ders., Marialis cultus, 2. Februar 1974; Johannes Paul II., Rosarium Viginis Mariae, 16. Oktober 2002.

27 Vgl. DVL 201. Die Clausula als Einschub wird in besonderer Weise empfohlen.

28 Vgl. DVL 198; Vgl. Benediktionale, Studienausgabe, hrsg. von der Internationalen Arbeitsgemeinschaft der Liturgischen Kommissionen im Deutschen Sprachgebiet, Segnung eines Rosenkranzes, Freiburg 1978, 215-218.

${ }^{29}$ Vgl. HdA $17 \S 1,1^{\circ}-2^{\circ}$.

${ }^{30}$ Vgl. HdA $17 \S 1,2^{\circ}$. 
c) Empfohlen werden außerdem im Direktorium über die Volksfrömmigkeit und die Liturgie auch Marianischen Litaneien. Grundsätzlich handelt es sich hierbei um Reihen von Anrufungen der Jungfrau Maria, die durch den steten Wechsel von Lob und Bitte gekennzeichnet sind.

Von herausragender Bedeutung ist die Lauretanische Litanei $^{31}$, die 1587 von Papst Sixtus V. approbiert und am 6. September 1601 von Papst Clemens VIII. durch das Dekret Quoniam multi des Heiligen Offiziums für die liturgische Verwendung genehmigt wurde. ${ }^{32}$ Die lobpreisenden Anrufungen Mariens als Mutter und als Jungfrau bzw. die Anrufungen Mariens in Bildern basieren zumeist auf Worten der Heiligen Schrift. ${ }^{33}$ Die Anrufungen Mariens in ihrer Mittlerrolle fußen im biblischen Grundgedanken, dass Maria die neue Eva ist, die alle seit der ersten Eva entstandenen Drangsale und Schäden beheben möge. Die Anrufungen Mariens als Königin basieren in der seit dem 6. Jahrhundert belegten Überlieferung der Kirche bzw. in den vom Lehramt vorgelegten marianischen Dogmen. ${ }^{34}$ Im Laufe der Zeit wurden der ursprünglichen Form weitere Anrufungen hinzugefügt. ${ }^{35}$ Eingebettet ist der marianische Lobpreis in die

31 „Der Text der Lauretanischen Litanei wurde zum ersten Mal im Rituale Romanum, im Anhang, in der Edition des Jahres 1874 eingefügt" (DVL 203, Fußnote 246). Sowohl im Missale Romanum wie im Pontifikale Romanum und im Rituale Romanum, die allesamt nach dem Zweiten Vatikanischen Konzil neu herausgegeben worden sind, findet sich die Lauretanische Litanei nicht mehr. Die ursprüngliche Fassung der Lauretanischen Litanei, die einer Pariser Handschrift um 1200 entstammt, wurde durch die Päpste kontinuierlich erweitert.

32 Vgl. B. Fischer, Litanei. 3. Anrufungslitanei, in: LTHK3, Bd. 6, 955.

33 Vgl. W. Dürig, Die Lauretanische Litanei, Sankt Ottilien 1990, 33-55.

34 Vgl. ebd., 65ff.

35 Vor der Approbation durch Papst Sixtus V. fügt Papst Pius V. im Jahre 1571 bereits die Anrufung „Hilfe der Christen“ der Lauretanischen Litanei hinzu. Nach der Approbation wurde es den Rosenkranzbruderschaften ab 1675 gestattet, die Anrufung „Königin des heiligen Rosenkranzes“ in der Litanei zu gebrauchen. Papst Leo XIII. ordnete 
Anrufung der Heiligen Dreifaltigkeit am Anfang der Litanei und die Anrufung Jesu Christi im Kyrie und Christe eleison wie im Agnus Dei vor der Abschlussoration.

Ebenfalls als Litanei empfohlen wird die Litanei für den Ritus der Krönung eines Bildes der seligen Jungfrau Maria, die als Alternative zur Fassung der Lauretanischen Litanei verstanden wird. ${ }^{36}$ Die besondere Wertschätzung gegenüber der Verwendung der Litaneien zu Ehren der seligen Jungfrau Maria kommt auch durch die Gewährung eines vollkommenen Ablasses zum Ausdruck. ${ }^{37}$

d) Der Hymnus Akathistos, der der byzantinischen Tradition entnommen ist und aus dem 7. Jahrhundert stammt, ist „eine der höchsten Ausdrucksformen marianischer Frömmigkeit“"

diese Anrufung durch das Apostolische Schreiben Salutaris ille vom 24. Dezember 1883 für die ganze Kirche verbindlich im Text der Litanei an. 1768 fügte Papst Clemens XIII. auf Bitten des spanischen Königs Karls III. die Anrufung „Mutter ohne Makel“ ein. Nach der Verkündigung des Dogmas der „ohne Erbsünde empfangenen Jungfrau“ im Jahr 1854 kam die Anrufung „Königin, ohne Erbsünde empfangen“ hinzu. Papst Leo XIII. nahm 1903 die Anrufung „Mutter vom guten Rat" in die Litanei auf. Papst Benedikt XV. ordnete am 5. Mai 1917 die Anrufung „Königin des Friedens“ an. Papst Pius XII. erweiterte nach der Verkündigung des Dogmas von der leiblichen Aufnahme Mariens in den Himmel die Litanei durch die Anrufung „Königin, in den Himmel aufgenommen“. Am 13. März 1980 ordnete Papst Johannes Paul II. an (vgl. CCDDS: Prot. N. CD 700/80), die Anrufung „Mutter der Kirche“ einzufügen. Derselbe Papst schrieb außerdem durch die Kongregation für den Gottesdienst und die Sakramentenordnung am 31. Dezember 1995 die Anrufung „Königin der Familien“ (vgl. Prot. N. 2500/95/L, in: Notitiae 32 (1996), 189-190) und im Jahr 2000 die Anrufung „Mutter der Barmherzigkeit" in die Lauretanische Litanei ein. Vgl. C. Maggioni, Regina Familiae, ora pro nobis, in: Notitiae 32 (1996), 207-216.

36 Vgl. DVL 203; vgl. Die Feier der Krönung eines Marienbildes. Studienausga$b e$, hrsg. von der Internationalen Arbeitsgemeinschaft der Liturgischen Kommissionen im Deutschen Sprachgebiet, Freiburg 1990, Nr. 41.

37 Vgl. HdA 22, $2^{\circ}$. 
(DLV 207). ${ }^{38}$ Im Direktorium wird er im Blick auf seine Quellen hin beschrieben; damit kommt zugleich der theologische Charakter zum Ausdruck. In DLV 207 heißt es: „Die Quellen, die diesen Hymnus inspiriert haben, sind: die Heilige Schrift, die auf den Ökumenischen Konzilien von Nizäa (325), Ephesus (431) und Chalkedon (451) definierte Lehre sowie die Lehren der Kirchenväter des Ostens im vierten und fünften Jahrhundert." In der jüngeren Kirchengeschichte hat der Hymnus Akathistos auch Eingang in den Cultus Marialis der Lateinischen Kirche gefunden ${ }^{39}$; das Gebet desselben ist ebenfalls mit der Gewährung eines Ablasses verbunden. ${ }^{40}$

e) Empfohlen wird auch die marianische Andachtsübung der Weihe an die Gottesmutter als „vertrauensvolle Hingabe an Maria" (DLV 204). Diese Form kann als individueller bzw. als gemeinschaftlicher Akt erfolgen. Im Direktorium wird dazu ausgeführt, dass „im Licht des Testaments Christi (vgl. Joh 19,25-27) [...] der Akt der ,Weihe' eine bewusste Würdigung der einzigartigen Stellung [ist], die Maria von Nazareth im Geheimnis Christi und der Kirche innehat“. Somit ist es „ein Akt des Vertrauens auf ihre Fürsprache und die Wirksamkeit ihres

38 Vgl. E.M. Zumbroich, Das Geheimnis der Gottesmutter - Hymnos Akathistos, Gaildorf 1983.

39 Im Direktorium werden in Fußnote 262 folgende Feierlichkeiten von Papst Johannes Paul II. angeführt, bei denen der Hymnus gesungen wurde: „Mit dem Gesang des Akatisthos in der Basilika von Groß St. Marien am 7. Juni 1981 wurde der Jahrestage der Konzilien von Konstantinopel I (381) und von Ephesus (431) gedacht. Der Hymnus wurde auch anlässlich des 450. Jahrestages der Erscheinung der Jungfrau Maria in Mexiko, vom 10. bis 12. Dezember 1981, gesungen. Während des Marianischen Jahres stand Johannes Paul II. in der Kirche S. Maria sopra Minerva der Matutin mit dem Akatisthos im byzantinisch-slawischen Ritus vor. Der Akatisthos [...] war der Anlass einer feierlichen vom Papst am 8. Dezember 2000 geleiteten Feier in der Basilika Groß St. Marien in Rom, zusammen mit Vertretern verschiedener byzantinisch-katholischer Kirchen".

${ }^{40}$ Vgl. HdA, 23, §1. 
Schutzes, auf ihre vielfältigen mütterlichen Eigenschaften, die sie als echte Mutter aller ihrer Kinder in der Gnadenordnung innehat". Trinitätstheologisch, christologisch, pneumatologisch und ekklesiologisch drückt sich dies in der liturgischen Formel wie folgt aus: „zum Vater durch Jesus Christus im Heiligen Geist, auf die Fürsprache Marias, [...] um das eigene Taufversprechen treu einzuhalten" (DVL 204). In besonderer Weise entfaltet der hl. Ludwig Maria Grignion de Montfort diese Andachtsform in seinem Werk „Das Goldene Buch der vollkommenen Hingabe an Maria". ${ }^{41}$ Darin leitet er zur Weihe an Christus durch die Hände Marias an, um die Taufverpflichtungen treu zu leben..$^{42}$ Die gemeinschaftliche Form drückt sich in ausgezeichneter Weise in der Akten der Hingabe an Maria aus, die die Päpste im Namen der ganzen Kirche vollzogen haben. ${ }^{43}$

${ }^{41}$ Vgl. Ludwig Maria Grignon de Montfort, Das Goldene Buch: Die Wahre Andacht zu Maria, das Geheimnis Mariä und die Liebe zum Kreuz, Feldkirch 2014. Vgl. F. Opitz, Marienweihe, Geschichte, Theologie, Probleme, Praxis, Beispiele, Münster ${ }^{3} 1993$.

42 Vgl. DVL 204; vgl. Johannes Paul II., Redemptoris Mater, 25. März 1987, 48. (Kurzform: RM)

${ }^{43}$ Als Beispiele werden angeführt: Weihe der Welt und des Menschengeschlechts an das unbefleckte Herz Mariens durch Papst Pius XII., vollzogen in Rom am 31. Oktober 1942, in: AAS 34 (1942), 345-346. Weihe Russlands an das unbefleckte Herz Mariens durch Papst Pius XII., vollzogen in Rom am 7. Juli 1952, in: AAS 44 (1952), 505-511; besonders 511. Weiheakt durch Papst Paul VI. an die Gottesmutter, vollzogen in Rom am 21. November 1964, in: AAS 56 (1964), 1017. Weihe der Welt an das unbefleckte Herz Mariens durch Papst Johannes Paul II., vollzogen in Tschenstochau am 4. Juni 1979 (Abgerufen am 20.7.2019: https://w2.vatican.va/content/john-paul-ii/it/homilies/ 1979/documents/hf_jp-ii_hom_19790604_polonia-jasna-gora.html). Weihe Österreichs an Maria durch Papst Johannes Paul II., vollzogen in Mariazell am 13. September 1983 (Abgerufen am 20.07.2019: http:// w2.vatican.va/content/john-paul-ii/de/speeches/1983/september/documents/hf_jp-ii_spe_19830913_preghieraver gine.html). Weiheakt durch Papst Johannes Paul II. an die Gottesmutter, vollzogen in Rom am 25. März 1984, in: AAS 1984, 61-64. Weihe aller Familien an Maria 
f) Außerdem wird im Direktorium auch auf die durch die Jahrhunderte empfohlene Praxis des Gebrauchs des Skapuliers, vor allem des Skapuliers der seligen Jungfrau vom Berge Karmel hingewiesen. ${ }^{44}$ Durch das Skapulier kommt die vertrauensvolle Hingabe an Maria zum Ausdruck, wie sie sich in der Marienweihe vollzieht. Im Ritus der Überreichung des Skapuliers wird unmittelbar Bezug auf das Taufversprechen genommen und erklärt, dass die Träger eifrig bestrebt sind, sich mit Hilfe der jungfräulichen Mutter Maria und zum Lobe der Dreifaltigkeit dem fleischgewordenen Wort gleichförmig zu machen, damit der Träger zum himmlischen Vaterland gelange..$^{45}$

g) Ebenso wie das Skapulier werden auch die marianischen Medaillen, vor allem die „wundertätige Medaille“ als Ausdruck des Cultus Marialis genannt. Im Direktorium wird mit Bezug auf das Rituale Romanum ausgeführt, dass diese dazu dienen, „die Liebe Gottes in Erinnerung zu rufen und das Vertrauen auf die selige Jungfrau Maria zu vermehren" (DLV 206).

h) Vergessen werden dürfen sicherlich nicht, da sie im Bereich der Volksfrömmigkeit ein verbreiteter Ausdruck des Cultus Marialis sind, die Preces ad Beatissimam Virginem Mariam, die selbst nicht im Direktorium enthalten sind. Unter diese fallen

durch Papst Johannes Paul II., vollzogen in Nazareth am 25. März 2000 (Abgerufen am 20.07.2019 http://w2.vatican.va/content/john-paul-ii/de/homilies/2000/documents/hf_jp-ii_hom_20000 325_nazareth. html). Akt der Weihe und des Anvertrauens an das unbefleckte Herz Mariens durch Papst Benedikt XVI., vollzogen in Fatima am 12 Mai 2010 (Abgerufen 20.07.2019: http://w2.vatican.va/content/benedict-xvi/de/prayers/documents/hf_ben-xvi_20100512_affidamento-fatima.html.) Akt der Weihe durch Papst Franziskus an die Gottesmutter, vollzogen in Rom am 13. Oktober 2013 (Abgerufen 20.07.2019: http:// w2.vatican.va/content/francesco/de/homilies/2013/documents/papa-francesco _20131013_omelia-giornata-mariana.html.

${ }^{44}$ Vgl. DLV 205; vgl. G. J. Weisensee, Das Kleid vom Himmel. Einführung in die Wissenschaft des Skapuliertragens, Lauerz ${ }^{3} 2003$.

${ }^{45}$ Vgl. DLV 205. 
das Magnificat sowie beispielsweise die Gebete Maria, Mater gratia; Memorare, o piissima Virgo Maria; Salve, Regina; Sancta Maria, succurre miseris oder auch das Sub tuum praesidium. Auch für diese gewährt die Kirche einen Teilablass. ${ }^{46}$

i) Ebenfalls keine Erwähnung im Direktorium über die Volksfrömmigkeit und die Liturgie finden die Wallfahrten zu den Marianischen Heiligtümern und die Wallfahrtsstätten selbst, die ein starker Ausdruck des Cultus Marialis sind. ${ }^{47}$ In der Enzyklika Redemptoris Mater stellt Papst Johannes Paul II. heraus: „Zu Recht hat das christliche Volk in seiner Frömmigkeit immer eine tiefe Verbindung zwischen der Verehrung der heiligen Jungfrau und dem Kult der Eucharistie gesehen: Dies ist eine Tatsache, die [...] in der Pastoral der marianischen Wallfahrtsorte ersichtlich ist. Maria führt die Gläubigen zur Eucharistie" (RM 44). Die besondere Wertschätzung des Apostolischen Stuhls kommt dadurch zum Ausdruck, dass für eine Wallfahrt zu internationalen, nationalen und diözesanen Heiligtümern, zu denen ja auch die marianischen gehören, ein vollkommener Ablass gewährt wird. ${ }^{48}$

Blickt man auf das bisher Ausgeführte zurück, so zeigt sich, wie vielfältig und zahlreich sich in der Liturgie und in den vom Lehramt der Kirche empfohlenen Andachtsübungen der Cultus Marialis ausdrückt.

\section{Das Zweite Vatikanische Konzil und die dogmatische Konstitution Lumen gentium}

Die Marienverehrung der Gegenwart und ihre inhaltlich durch die Kirche bestimmte Form kann man nur verstehen, wenn man

${ }^{46} \mathrm{Vgl} . \mathrm{HdA} 17 \S 2,1^{\circ} ; 3^{\circ}$.

47 Vgl. Kongregation für den Gottesdienst und die Sakramentenordnung, Orientamenti e proposte per la celebrazione dell'Anno Mariano (Prot. N. 401/87), in: Notitiae 23(1987), 342-396, besonders Nrn. 75-79. ${ }^{48} \mathrm{Vgl}$. HdA $33 \S 1,4^{\circ}$. 
das zentrale Ereignis des Zweiten Vatikanischen Konzils in den Blick nimmt. Auf dem Konzil rang man nicht um die Frage, um ein Mehr oder Weniger an Marienverehrung, sondern darum, wie man den innersten Sinn der Stellung und der Verehrung Mariens bestmöglich zum Ausdruck bringen kann. Sollte man „Maria als Gestalt für sich, gewissermaßen auf eigenem Podest mit der Gefahr der Isolierung [behandeln], oder dadurch, dass man von Maria in jenen Zusammenhängen spricht, die ihr erst ihre Bedeutung im Heilswerk und damit in der Verehrung der Kirche gegeben haben" ${ }^{49}$ ? Die Väter des Konzils stellten heraus, dass Jesus Christus durch Maria in die Geschichte eingetreten ist, um die Menschheit durch sein Erlösungswerk aus der Knechtschaft der Unheilsgeschichte zu befreien, und dass der Sohn Gottes selbst die Kirche der Geschichte eingestiftet hat. Damit muss die Kirche ebenso marianisch bestimmt sein, wie Jesus Christus selbst und sein Erlösungswerk marianisch bestimmt sind.$^{50}$ Aufgrund dieser Bestimmung behandelten die Väter das Thema „Maria“ nicht in einem eigenen Dokument, sondern fügten es im achten Kapitel der dogmatischen Konstitution über die Kirche Lumen Gentium ein..$^{51}$ Die Abstimmung zu dieser Entscheidung wird als „historische Wende ${ }^{\text {"52 }}$ bzw. als "geistige Wasserscheide "53 bezeichnet, da sie dazu führte, einen neuen mariologischen Ansatz zu erarbeiten, der auch für die Marienfrömmigkeit Folgen haben sollte. In LG 55-65 wird die Gegenwart Mariens in der Heilsgeschichte aufgezeigt und so in das

${ }_{49}$ O. Semmelroth, Kommentar zum Achten Kapitel der Dogmatischen Konstitution über die Kirche, (=LTHK2, Das Zweite Vatikanische Konzil, Teil I), 323.

50 Vgl. ebd., 328.

51 Vgl. Zweites Vatikanisches Konzil, Lumen gentium, in: AAS 57 (1965), 58-64.

52 S. De Fiores, Maria in der Geschichte von Theologie und Frömmigkeit, in: HdM2, Bd. 1, 240. (Kurzform: De Fiores)

53 J. Ratzinger und H. U. Von Baltasar, Maria - Kirche im Ursprung, Freiburg 1980, 18. 
Geheimnis Christi und der Kirche eingefügt. ${ }^{54}$ Damit verbindet sich, dass nach der inneren Logik der Heilsgeschichte, die Verehrung Mariens mit „seismographischer Empfindlichkeit “55 den Glauben der Christen anzeigt. Es werden damit nicht Privilegien Mariens angesammelt und systematische Konklusionen angehäuft, die immer zu einer Isolierung Mariens geführt haben, sondern das Dasein Mariens wird in der „Lebensmitte der Heilsgeschichte ${ }^{456}$ aufgezeigt. ${ }^{57}$ Seit Beginn des Christusglaubens gilt nämlich: „Im gleichen Moment, da sich die Christen des wahren Wesens ihres Herrn bewusst wurden, richtet sich ihr Blick auch auf Maria“. ${ }^{58}$ So wird sie "gleichzeitig ein Zentrum der Einheit und ein Brennpunkt des Widerscheins der göttlichen Geheimnisse". 59

Durch die Einordnung Mariens in Lumen gentium in die Kirchen bzw. ihre Zuordnung zur Kirche verhindert das Konzil eine erneute Überhöhung Mariens, wie sie sich in der Kirchengeschichte oftmals ereignet hat. Das achte Kapitel von Lumen gentium legt somit oberflächlich betrachtet eine ekklesiotypische Perspektive im Blick auf Maria nahe und bedingt einem marianischen Minimalismus bezüglich des Cultus Marialis. Jedoch ist, wie sich in LG 55-65 deutlich zeigt, die christotypische Perspektive zentral und bestimmend, die eine maximale Ausdehnung der marianischen Frömmigkeit ermöglicht. Zusammenfassend heißt es in LG 66, dass in der Ehrung der Mutter der Sohn richtig erkannt, geliebt und verherrlicht wird und seine Gebote beobachtet werden. Dies öffnet den Weg zu einer maximalen Ausdeh-

54 Vgl. De Fiores, 244.

55 Beinert, 7.

56 Vgl. De Fiores, 244.

57 Vgl. G. Greshake, Maria - Ecclesia. Perspektiven einer marianisch grundierten Theologie und Kirchenpraxis, Regensburg 2014, $183 f$.

58 Beinert, 6.

59 S. De Fiores, Maria nel mistero di Cristo e della Chiesa. Commento al capitolo mariano del Concilio Vaticano II, Roma 31984, 141 (Hervorhebungen im Original). 
nung des Cultus Marialis. Treffend stellen die Väter des Konzils fest: „Maria wird, durch Gottes Gnade nach Christus, aber vor allen Engeln und Menschen erhöht, mit Recht, da sie ja die heilige Mutter Gottes ist und in die Mysterien Christi einbezogen war, von der Kirche in einem Kult eigener Art geehrt" (LG 66). Und das Konzil „mahnt [...] alle Kinder der Kirche, die Verehrung, vor allem die liturgische, der seligen Jungfrau großmütig zu fördern, die Gebräuche und Übungen der Andacht zu ihr, die im Laufe der Jahrhunderte vom Lehramt empfohlen wurden, hochzuschätzen und das, was in früherer Zeit über die Verehrung der Bilder Christi, der seligen Jungfrau und der Heiligen festgesetzt wurde, ehrfürchtig zu bewahren“ (LG 67). Außerdem wird betont: „Die Gläubigen aber sollen eingedenk sein, dass die wahre Andacht weder in unfruchtbarem und vorübergehendem Gefühl noch in irgendwelcher Leichtgläubigkeit besteht, sondern aus dem wahren Glauben hervorgeht, durch den wir zur Anerkennung der Erhabenheit der Gottesmutter geführt und zur kindlichen Liebe zu unserer Mutter und zur Nachahmung ihrer Tugenden angetrieben werden" (LG 67).

\section{Papst Paul VI und die Enzyklika Marialis Cultus}

Die Enzyklika Marialis Cultus, von Papst Paul VI. am 2. Februar 1974 unterzeichnet, ist als Fortführung und Explizierung der marianischen Linie des Zweiten Vatikanischen Konzils zu verstehen. In diesem Dokument geht der Papst zunächst näher auf die Marienverehrung in der Liturgie ein. Nach den in der Liturgie enthaltenen Maßstäben über den Rang und die Stellung Mariens hebt er im Mittelteil der Enzyklika, die Prinzipien und Direktiven für die Marienverehrung hervor. Im Abschließenden Teil III betrachtet er näher das Angelus- und das Rosenkranzgebet. ${ }^{60}$

${ }^{60}$ Vgl. Beinert, $10 f$. 
Grundsätzlich stellt Paul VI. heraus, dass der Cultus Marialis nur als Teil der Christusverehrung sinnvoll und als solcher auf diese ausgerichtet christlich ist. Insofern muss die Marienverehrung sich "in harmonischer Unterordnung unter die Christusverehrung" (MC Einführung) entfalten und um sie herum kreisen wie um "ihren natürlichen und notwendigen Mittelpunkt“ (MC Einführung). Deutlich wird dies in MC 2-15 dargelegt, indem die erneuerte römische Liturgie anhand des Calendarium Romanum in den Blick genommen wird. „Was sich gegenüber der vorkonziliaren Liturgie [...] geändert hat, [...] ist [...] der Blickwinkel, unter dem sie gefeiert wird“. ${ }^{61}$ In Marialis Cultus stellt der Papst deshalb fest: „die nachkonziliare Erneuerung hat die selige Jungfrau Maria [...] in entsprechender Sicht im Zusammenhang mit dem Geheimnis Christi betrachtet und ihr im Einklang mit der Überlieferung die einzigartige Stellung zuerkannt, die ihr als heilige Gottesgebärerin und erhabene Gefährtin des Erlösers zukommt" (MC 15).

Im Mittelteil der Enzyklika Marialis Cultus geht der Paul VI. näher auf die inhaltliche Bestimmung des Cultus Marialis ein. Er hebt hervor, dass [a] „die Andachtsübung zur seligen Jungfrau Maria den trinitarischen und christologischen Charakter zum Ausdruck bringen muss" (MC 25). Dies begründet er damit, dass der eine christliche Kult jener Akt ist, der "dem Vater durch Christus im Heiligen Geist" (MC 25) dargebracht wird. Näher charakterisiert Paul VI. dies im Blick auf Maria, wenn er ausführt: „Bei Maria ist alles auf Christus hin bezogen und von ihm abhängig: im Hinblick auf ihn hat sie Gottvater von aller Ewigkeit her als ganz heilige Mutter erwählt und sie mit den Gaben des Heiligen Geistes ausgestattet" (MC 25). Von daher muss im Cultus Marialis [b] die christologische Dimension besonders hervortreten. [c] Biblisch muss sie sein, da die Bibel „auf wunderbare Weise den Heilsplan Gottes für die Menschen darstellt“ (MC 30) und „als Ganzes vom Mysterium des Erlö-

${ }^{61}$ Ebd., 14. 
sers durchdrungen ist“ (MC 30). [d] Liturgisch geprägt muss der Marialis Cultus sein, da die Liturgie „die goldene Norm für die christliche Frömmigkeit" (MC 23) ist. ${ }^{62}$ Außerdem muss der Cultus Marialis sowohl [e] ekklesiologischen wie ökumenischen Charakter besitzen, da „es dem echten Kult der Seligen Jungfrau wesentlich eigen ist, dass ,in der Verehrung der Mutter der Sohn [...] richtig erkannt, geliebt und verherrlicht wird, [...] der Quelle und [...] Mittelpunkt der kirchlichen Gemeinschaft" (MC 32) ist. Schließlich muss der Cultus Marialis sich auch durch seinen [f] anthropologischen Charakter auszeichnen: Die Jungfrau Maria ist nämlich von der Kirche den Gläubigen zur Nachahmung empfohlen, „weil sie in ihren konkreten Lebensbedingungen vorbehaltlos und verantwortungsbewusst dem Willen Gottes Folge geleistet hat (vgl. Lk 1,38); weil sie von ihm das Wort entgegennahm und in die Praxis umsetzte; weil ihr Handeln von der Liebe und der Bereitschaft zum Dienen beseelt war; weil sie die erste und vollkommenste Jüngerin Christi gewesen ist" (MC 35).

Im dritten Teil der Enzyklika Marialis Cultus geht Papst Paul VI. schließlich auf das Angelus- und Rosenkranzgebet ein. Der Angelus bedarf, so stellt der Papst grundlegend heraus, „keiner Erneuerung“ (MC 41). Er versteht das Angelusgebet als besonderen Ausdruck des Cultus Marialis, da es gemäß seiner theologischen Struktur ,als Parallel- und Kurzform des Rosenkranzes"63 zu verstehen ist. Auf das Rosenkranzgebet geht der Papst näher in den Artikeln 42-52 ein. Von zentraler Bedeutung ist, dass Paul VI. den biblischen Charakter herausstellt, da die hauptsächliche Form dieses Gebetes biblisch ist und die Geheimnisse des Rosenkranzes dem Evangelium entstammen. „Der Rosenkranz betrachtet [...] in harmonischer Folge die wichtigsten Heilsereignisse, die sich in Christus zugetragen haben: von der jungfräulichen Empfängnis und den Geheimnissen seiner Kindheit bis zu dem erhabenen Geschehen von Ostern - des

${ }^{62}$ Vgl. auch MC 31.

${ }^{63}$ F. Courth, Marianische Gebetsformen, in: HdM2, Bd. 1, 548. 
seligen Leidens und der glorreichen Auferstehung - und weiter bis zu deren Auswirkung auf die bildende Kirche am Pfingsttag und auf die Heilige Jungfrau an dem Tage, an dem sie nach Beendigung ihres Erdenlebens mit Leib und Seele in die himmlische Heimat aufgenommen wurde" (MC 45). Daraus folgt, dass der Rosenkranz als biblisches Gebet, „zutiefst christologisch geprägt“ (MC 46) ist. Sodann ist der Rosenkranz von seinem Ursprung her als "Psalter der Jungfrau“ (MC 48) ein liturgisches Gebet, welches das Herrengebet ebenso wie die Doxologie durch das Gloria Patri beinhaltet. ${ }^{64}$ Schließlich stellt der Papst durch die Hervorhebung des Rosenkranzgebetes in der Familie den ekklesiologischen Charakter dieses Gebetes heraus. Denn die „christliche Familie ist [...] eine Art Hauskirche, wenn ihre Glieder [...] sich in den liturgischen Kult eingliedern" (MC 52).

Aus dem in der Enzyklika Marialis Cultus Ausgeführten geht hervor: „Die Andacht zur Seligen Jungfrau in der Kirche ist ein wesentlicher Bestandteil des christlichen Kultes" (MC 56). Zugleich zeigt sich: „Die Verehrung, die die Kirche an allen Orten zu allen Zeiten der Gottesmutter erwiesen hat [...], ist ein eindrucksvolles Zeugnis ihrer lex orandi und eine Einladung, in den Herzen ihre lex credendi neu zu beleben. Umgekehrt verlangt die lex credendi der Kirche, dass überall ihre lex orandi im Hinblick auf die Mutter Christi lebendig aufblühe. Ein solcher Marienkult ist tief im offenbarten Gotteswort verwurzelt und hat solide dogmatische Grundlagen“ (MC 56).

\section{Papst Johannes Paul II. und das Apostolische Schreiben Rosarium Virginis Mariae}

Was das Zweite Vatikanische Konzil im Blick auf die Marienverehrung grundgelegt hat, erläutert der heilige Papst Paul VI.

${ }^{64}$ Vgl. MC 49. 
in der Enzyklika Marialis Cultus. Die Kongregation für den Gottesdienst und die Sakramentenordnung veröffentlichte dann in der Folge, während des Pontifikates des heiligen Papst Johannes Paul II., wie eingangs vorgestellt, das Direktorium über die Liturgie und die Volksfrömmigkeit. Hier wird der Cultus Marialis am Beginn des dritten Jahrtausends vor allem beschrieben und für die Gegenwart umfänglich definiert. Papst Johannes Paul II. selbst setzt innerhalb seines langen und segensreichen Pontifikates zahlreiche Akzente im Blick auf den Cultus Marialis. Von besonderer Bedeutung ist das am 16. Oktober 2002 von ihm unterzeichnete Apostolische Schreiben Rosarium Virginis Mariae. ${ }^{65}$ In diesem Dokument vollzieht er einen wesentlichen und weiterführenden Schritt für den Cultus Marialis. Mit päpstlicher Autorität schließt er die seit der Approbation des Rosenkranzes bestehende inhaltliche Lücke, die im Gebet des Rosenkranzes offiziell besteht. In RVM 19 sagt der Papst im Blick auf das Rosenkranzgebet: „Um den christologischen Gehalt dieses Gebetes deutlicher zu machen, halte ich es für angebracht, eine angemessene Ergänzung vorzunehmen, die auch die Geheimnisse des öffentlichen Lebens zwischen der Taufe und dem Leidensweg Christi einbezieht". Als die fünf bedeutenden Momente benennt Papst Johannes Paul II. als erstes die Taufe Jesu im Jordan, dann die Selbstoffenbarung Jesu bei der Hochzeit zu Kana, als drittes Jesu Verkündigung des Reiches Gottes mit dem Ruf zur Umkehr, als viertes die Verklärung Jesu auf dem Berg Tabor und schließlich als fünftes die Einsetzung der Eucharistie durch Jesus als sakramentaler Ausdruck des Ostergeheimnisses. ${ }^{66}$ Durch diese Einfügung der bedeutenden Momente des öffentlichen Lebens Jesu, wird der Rosenkranz in einem umfassenden Sinn des Wortes noch mehr zum „Kompendium des Evangeliums“ (RVM 19).

\footnotetext{
65 Johannes Paul II., Rosarium Virginis Mariae, 16. Oktober 2002 (VApS 156), Bonn 2002. (Kurzform: RVM)

66 Vgl. RVM 21.
} 
Zugleich stellt Papst Johannes Paul II. das Rosenkranzgebet als besondere Ausdrucksform des Cultus Marialis heraus. Dies geschieht nicht zuerst auf Grund der Tatsache, dass der Papst bereits am Beginn seines Pontifikates bekennt: „Der Rosenkranz ist mein Lieblingsgebet" (RVM Einführung). ${ }^{67}$ Sondern die Hervorhebung des Rosenkranzgebetes erfolgt auf Grund der christologischen Bedeutung. Hierzu muss Folgendes bedacht werden: Johannes Paul II. stellt fest, dass die wesentliche christliche Aufgabe jedes Jüngers Christi darin besteht, „die Augen auf das Antlitz Christi gerichtet zu halten“ (RVM 9). Denn „im Betrachten des Angesichts öffnen wir uns, um das Geheimnis des dreifaltigen Lebens in uns aufzunehmen" (RVM 9). Dieses Betrachten versteht der Papst im biblischen Sinn von Gedächtnis (zakar), denn es vollzieht sich die Gegenwärtigsetzung der Werke, "die Gott in der Heilsgeschichte erfüllt hat“ (RVM 13). „Diese Ereignisse sind nicht nur ein ,Gestern'; [sondern] zugleich sind sie das ,Heute 'der Erlösung" (RVM 13). Selbstverständlich ist dem Papst klar, dass die Aktualisierung der Heilswerke sich vor allem in der Liturgie vollzieht und nicht zuerst in der Volksfrömmigkeit. Jedoch betont er: „Wenn die Liturgie, das Handeln Christi und der Kirche, ein Heilswerk par excellence darstellt, dann ist der Rosenkranz als eine Meditation über Christus mit Maria Heilsbetrachtung" (RVM 13). Die besondere Bedeutung Mariens kommt in dieser Heilsbetrachtung daher, dass Maria unaufhörlich mit den Augen auf Christus gerichtet lebt. Auch nach ihren Erdentagen ändert sich dies nicht. In der Herrlichkeit des Himmels lebend, legt sie „den Gläubigen [...] unaufhörlich die ,Geheimnisse ihres Sohnes vor“, nämlich die „Erinnerungen an Jesus, die sich ihrer Seele einprägten" (RVM 11). Im Blick auf die außerordentliche Bedeutung Mariens stellt Papst Johannes Paul II. fest: „Niemand hat sich mehr als Maria der Betrachtung des Antlitzes Christi mit gleicher Beharrlichkeit hingegeben" (RVM 10). So empfiehlt

67 Vgl. Johannes Paul II., Angelus am 29. Oktober 1978, in: OR dt., 44 (1978), 3. 
der Papst das Rosenkranzgebet als vorzüglichen Ausdruck des Cultus Marialis den Gläubigen, denn „das Gehen durch die Szenen des Rosenkranzes an der Seite Mariens bedeutet, sich ,in die Schule Mariens' zu begeben, um Christus zu erfassen und um in die Geheimnisse einzudringen" (RVM 14). In diesem Erfassen und Eindringen vollzieht sich jedoch nach den Worten des Papstes noch mehr: Denn „während des geistlichen Vollzugs des Rosenkranzes, der - in Gemeinschaft mit Maria - auf der unaufhörlichen Betrachtung des Antlitzes Christi gründet, erreicht man dieses anspruchsvolle Ideal des Ähnlichwerdens mit Ihm" (RVM 15).

Eine legitime Ergänzung nach dem Direktorium über die Liturgie und die Volksfrömmigkeit und der Enzyklika Rosarium Virginis Mariae.

Nimmt man die vor allem biblisch, christologisch und liturgisch bestimmte Grundlinie des Marienverehrung auf, wie sie vom Zweiten Vatikanischen Konzils in Lumen gentium ansetzt, über Marialis Cultus von Papst Paul VI. zum Direktorium über die Liturgie und die Volksfrömmigkeit führt und dann in das Apostolische Schreiben Rosarium Virginis Mariae von Papst Johannes Paul II. mündet, so zeigt sich im Blick auf den Cultus Marialis in der Gegenwart eine legitime Möglichkeit einer besonderen Ausdrucksweise. Bereits im Direktorium über die Liturgie und die Volksfrömmigkeit heißt es bezüglich der Rosenkranzgesätze: „Es [ist] erlaubt, die Andachtsübung der liturgischen Zeit anzugleichen. So handeln zum Beispiel die Gläubigen korrekt, die am sechsten Januar, dem Fest der Erscheinung des Herrn, die freudenreichen Geheimnisse und als „fünftes Geheimnis“ die Anbetung der Könige anstelle des Wiederfindens des zwölfährigen Jesus im Tempel zu Jerusalem betrachten. Selbstverständlich muss dieser Austausch in Anlehnung an die Heilige Schrift und an das liturgische Eigengut ausgewogen gehandhabt werden" (DLV 200). Mit einem päpstlichen Akt hat ja Johannes Paul II. offiziell mit dem lichtreichen Geheimnis fünf bedeutende Momente 
des Christusmysteriums dem Rosenkranzgebet hinzugefügt. Den Gläubigen steht es für das persönliche wie gemeinschaftliche Gebet des Rosenkranzes zu, auf der Basis der Heiligen Schrift, unter Berücksichtigung der Liturgie und im Blick auf Jesus Christus im Rahmen des Cultus Marialis weitere Clausulae im Rosenkranzgebet fruchtbringend zu betrachten. Bereits 1940 macht dazu Romano Guardini in seinem Buch über den Rosenkranz den Vorschlag, anstelle der vierten und fünften Clausula des glorreichen Rosenkranzes die beiden Christusgeheimnisse zu betrachten: Jesus, „der wiederkommen wird in Herrlichkeit" ${ }^{\text {"88 }}$ und Jesus, „dessen Reich wird kein Ende sein “. ${ }^{69}$ Im Katholischen Gebet- und Gesangbuch Gotteslob ${ }^{70}$, das für die Diözesen Deutschlands und Österreichs wie Bozen-Brixen und Lüttich 1975 herausgegeben wurde, fand sich unter der Nr. 33,6 der bischöflich genehmigte trostreiche Rosenkranz, dessen Gesätze lauten: „der als König herrscht“, „der in seiner Kirche lebt und wirkt“, „der wiederkommen wird in Herrlichkeit“, "der richten wird die Lebenden und die Toten“, „der alles vollenden wird“. Bereits 1978 wurden weitere Vorschläge im deutschsprachigen Raum vorgelegt. Im Marienlob der Actio Mariae werden Rosenkranzgeheimnisse, die jeweils durch fünf Clausulae aufgeteilt sind und auf der Heiligen Schrift wie der Tradition beruhen, veröffentlicht; zu diesen zählt der Rosenkranz des verborgenen Lebens Jesu ${ }^{71}$ und der Rosenkranz des

68 R. Guardini, Der Rosenkranz Unserer Lieben Frau, 3. Taschenbuchauflage 1996, 82.

69 Ebd., 84.

70 Gotteslob, Katholischen Gebet- und Gesangbuch für die Diözesen Deutschlands und Österreichs wie Bozen-Brixen und Lüttich, hrsg. von den (Erz-) Bischöfen Deutschlands und Österreichs und dem Bischof von Bozen Brixen und Lüttich, Köln 1975.

${ }^{71}$ Marienlob, hrsg. von der Actio Mariae in Zusammenarbeit mit dem Institutum Marianum Regensburg, Abendsberg 1978, 130. (Kurzform: Marienlob) Der Rosenkranz des verborgenen Lebens Christi besteht aus den Clausulae „der seinen Eltern untertan war“, „der als Zimmermann 
öffentlichen Lebens Jesu. ${ }^{72}$ Bei genauerer Betrachtung zeigt sich, dass die Clausulae des verborgenen und öffentlichen Lebens in ihren wesentlichen Momenten bereits vorwegnehmen, was Papst Johannes Paul II. im lichtreichen Rosenkranz als Ergänzung vorgelegt hat. Außerdem werden im Marienlob weitere Rosenkranzgeheimnisse, die vom Kölner Pfarrer Msgr. Wolfram Krusenotto ${ }^{73}$ verfasst wurden, als Ergänzung vorgeschlagen: die vorbereitenden, die offenbarenden, die heilbringenden, die seligmachenden, die menschenfreundlichen, die mitleidenden, die apostolischen, die nachfolgenden, die verklärenden, die christologischen, die kostbaren, die eucharistischen, die endzeitlichen, die tröstenden und die frohmachenden Geheimnisse, wie auch die Geheimnisse der Verwerfung und die als Jesus-Geheimnisse bezeichneten. ${ }^{74}$ In diesen Ergänzungen verdeutlicht sich, dass der Rosenkranz "Gebet gewordenes Credo"75 ist und ihm im Cultus Marialis eine außerordentliche Bedeutung zukommt.

\section{Der Cultus Marialis für die Gegenwart}

Die in diesem Beitrag vorgelegte Analyse zeigt, dass das Direktorium über die Liturgie und die Volksfrömmigkeit das verbindliche Dokument für die Bestimmung des Cultus Marialis ist. In ihm werden im Blick auf die Liturgie die Räume für die Marienver-

die Arbeit geheiligt hat", „der im Jordan getauft wurde“, „der vom Teufel versucht wurde“, „der Jünger und Apostel berufen hat".

72 Ebd., 130f. Der Rosenkranz des öffentlichen Lebens Jesu setzt sich zusammen aus den Clausulae: „der die Frohe Botschaft vom Gottesreich verkündet hat", „der seine Herrlichkeit durch Wunder geoffenbart hat", „der Schmähungen und Widerspruch ertragen hat", „der den Seinen die Füße gewaschen hat", "der uns das Geheimnis der Eucharistie geschenkt hat".

${ }^{73}$ Vgl. ebd., 153.

74 Vgl. ebd., 149-153.

75 G.-M. Garrone, Maria gestern und heute, München 1978, 61. 
ehrung innerhalb des liturgischen Jahres aufgezeigt und die vom Lehramt besonders empfohlenen Andachtsübungen als Ausdruck der Marienverehrung benannt. Die dabei zugrundeliegenden Prinzipien basieren auf den Bestimmungen, die die Väter des Zweiten Vatikanischen Konzils, vor allem in den beiden Dokumenten Lumen gentium und Sacrosanctum concilium vorgenommen haben. Maria wird in der Mitte der Heilsgeschichte verortet und ihre Verehrung von daher bestimmt. Inhaltlich ausgeführt werden die Grundbestimmungen des Cultus Marialis durch die Enzyklika Marialis Cultus. In dieser stellt Papst Paul VI. deutlich heraus, dass die Marienverehrung vor allem durch eine biblisch, trinitarisch-christologische und ekklesiologische Grunddimension bestimmt sein muss, die liturgisch geprägt zu sein hat. So muss die Marienverehrung eingebettet sein im einen Kult der Christusverehrung. Papst Johannes Paul II. führt diese inhaltlich bestimmte Linie von Marialis Cultus fort, indem er die christologische Dimension der Marienverehrung in besonderer Weise akzentuiert. Marienverehrung vollzieht sich an der Seite Mariens und dient der Heilsbetrachtung als Vergegenwärtigung. Mit den Augen Mariens richtet sich der Blick auf ihren Sohn Jesus Christus, in der Betrachtung seines Heilswerkes ereignet sich das Ähnlichwerden mit dem Erlöser. In unüberbietbarer Weise verwirklicht sich dieses geistliche Geschehen im Großen im Gebet des Rosenkranzes und im Kleinen im Gebet des Angelus bzw. des Regina Caeli. Um das Rosenkranzgebet noch fruchtbringender werden zu lassen, hat Papst Johannes Paul II. die Ordnung der Clausulae des Rosenkranzes durch die fünf zentralen Momente des lichtreichen Geheimnisses ergänzt. Damit hat er nicht nur die inhaltliche Lücke in der Betrachtung der Heilsgeheimnisse offiziell geschlossen, sondern zugleich gezeigt, wie in verantwortlicher Weise die Betrachtung der Heilsgeheimnisse in Jesus Christus - im Ausdruck des Cultus Marialis - vertieft werden können. Einige Beispiele einer Ergänzung - auf der rechtlichen Grundlage des Direktoriums über die Liturgie und 
die Volksfrömmigkeit und unter Beachtung der inhaltlichen Bestimmungen von Marialis Cultus und Rosarium Virginis Mariae - zeigen, in welcher Form der Cultus Marialis heute noch fruchtbringender praktiziert werden kann. Maria wird um Jesu Christi willen verehrt, da sie die Gläubigen unaufhörlich zu ihrem Sohn und seinem Heilswerk führt. So trägt der Cultus Marialis dazu bei, mit Jesus Christus immer tiefer verbunden und ihm immer ähnlicher zu werden.

\section{Jak dzisiaj czcić Maryję?}

Niniejszy artykuł ukazuje drogę i warunki, jakie zostały wyznaczone współczesności przez Marialis cultus. Ta adhortacja zaś nawiązuje do II Soboru Watykańskiego i wypływa z niego, a także prowadzi przez główne wypowiedzi Pawła VI do pontyfikatu św. Jana Pawła II. Okazuje się przy tym, iż znajdujące się u podstaw odnowy kultu zasady opierają się na wyznacznikach konstytucji dogmatycznej Lumen gentium i znajdują zastosowanie w adhortacji Marialis cultus. Z kolei postanowienia Marialis cultus są wprowadzone do Dyrektorium do liturgii i ludowej pobożności. Papież Jan Paweł II kontynuuje je treściowo, kiedy chrystologiczne wymiary maryjnej czci akcentuje w szczególny sposób w swoim piśmie apostolskim Rosarium Virginis Mariae. Wypełnia on przy tym istniejący brak w porządku medytacyjnym różańca, wprowadzając pięć jego centralnych momentów poprzez tajemnice światła. Kilka dalszych przykładów względnego uzupełnienia dopełnia ciąg myśli powyższej prezentacji i pokazuje, w jakiej formie wskazania Marialis cultus mogą być owocne w praktyce także dzisiaj. 